\title{
GENERALIZED B-FREDHOLM BANACH ALGEBRA ELEMENTS
}

\author{
MILOŠ D. CVETKOVIĆ, ENRICO BOASSO, \\ AND SNEŽANA Č. ŽIVKOVIĆ-ZLATANOVIĆ
}

\begin{abstract}
Given a (not necessarily continuous) homomorphism between Banach algebras $\mathcal{T}: \mathcal{A} \rightarrow \mathcal{B}$, an element $a \in \mathcal{A}$ will be said to be B-Fredholm (respectively generalized B-Fredholm) relative to $\mathcal{T}$, if $\mathcal{T}(a) \in \mathcal{B}$ is Drazin invertible (respectively Koliha-Drazin invertible). In this article, the aforementioned elements will be characterized and their main properties will be studied. In addition, perturbation properties will be also considered.
\end{abstract}

\section{INTRODUCTION}

The Atkinson theorem states that necessary and sufficient for a Banach space operator to be Fredholm is that its coset in the Calkin algebra is invertible. This well known result led to the introduction of a Fredholm theory relative to a Banach algebra homomorphism, see [11]. This theory has been developed by many authors, which have also studied other classes of objects such that Weyl, Browder and Riesz Banach algebra elements relative to a (not necessarily continuous) homomorphism, see for example [11, 12, 13, 14, 20, 10, 21, 8, 15, 1, 30, 22, 31, 32].

Recall that the theory of Fredholm operators was generalized. In fact, the notion of B-Fredholm operator was introduced and studied, see section 2 or [2, 23]. In addition, given $\mathcal{X}$ a Banach space and $T \in \mathcal{L}(\mathcal{X})$ a bounded and linear map, according to [4, Theorem 3.4], $T$ is B-Fredholm if and only if $\tilde{\pi}(T) \in \mathcal{L}(\mathcal{X}) / \mathcal{F}(\mathcal{X})$ is Drazin invertible, where $\mathcal{F}(\mathcal{X})$ denotes the ideal of finite rank operators defined on $\mathcal{X}$ and $\tilde{\pi}: \mathcal{L}(\mathcal{X}) \rightarrow \mathcal{L}(\mathcal{X}) / \mathcal{F}(\mathcal{X})$ is the quotient homomorphism. This Atkinson-type theorem for B-Fredholm operators leads to the following definition. Let $\mathcal{A}$ and $\mathcal{B}$ be two complex unital Banach algebras and consider a (not necessarily continuous) homomorphism $\mathcal{T}: \mathcal{A} \rightarrow \mathcal{B}$. The element $a \in \mathcal{A}$ will be said to be B-Fredholm (respectively generalized B-Fredholm) relative to $\mathcal{T}$, if $\mathcal{T}(a) \in \mathcal{B}$ is Drazin invertible (respectively Koliha-Drazin invertible).

The main objective of this article is to study (generalized) B-Fredholm Banach algebra elements relative to a (not necessarily continuous) homomorphism. In section 3, after having recalled some preliminary facts in section 2, the aforementioned elements will be characterized and studied. What

2010 Mathematics Subject Classification. Primary 46H05, 47A10; Secondary 47A53, $47 \mathrm{~A} 55$.

Key words and phrases. Banach algebra, homomorphism, B-Fredholm element, generalized B-Fredholm element, perturbation class. 
is more, B-Weyl and B-Browder elements will be also considered. On the other hand, in section 4 perturbation properties of B-Fredholm elements will be studied and in section 5 perturbations of (generalized) B-Fredholm elements with equal spectral idempotents with respect to a (not necessarily continuous) homomorphism will be considered.

\section{Preliminary Definitions and Facts}

From now on $\mathcal{A}$ will denote a complex unital Banach algebra with identity 1 . Let $\mathcal{A}^{-1}, \mathcal{A}_{\text {left }}^{-1}, \mathcal{A}_{\text {right }}^{-1}, \mathcal{A}^{\bullet}$ and $\mathcal{A}^{\text {nil }}$ denote the set of all invertible elements in $\mathcal{A}$, the set of all left invertible elements in $\mathcal{A}$, the set of all right invertible elements in $\mathcal{A}$, the set of all idempotents in $\mathcal{A}$ and the set of all nilpotent elements in $\mathcal{A}$, respectively. Given $a \in \mathcal{A}, \sigma(a)$ and iso $\sigma(a)$ will stand for the spectrum and the set of isolated spectral points of $a \in \mathcal{A}$, respectively. Recall, if $K \subseteq \mathbb{C}$, then acc $K$ is the set of limit points of $K$ and iso $K=K \backslash$ acc $K$.

An element $a \in \mathcal{A}$ is said to be Drazin invertible, if there exists a necessarily unique $b \in \mathcal{A}$ and some $k \in \mathbb{N}$ such that

$$
b a b=b, \quad a b=b a, \quad a^{k} b a=a^{k} .
$$

If the Drazin inverse of $a$ exists, then it will be denoted by $a^{d}$. In addition, the index of $a$, which will be denoted by ind $(a)$, is the least non-negative integer $k$ for which the above equations hold. When ind $(a)=1$, $a$ will be said to be group invertible, and in this case its Drazin inverse will be referred as the group inverse of $a$; moreover, it will be denoted by $a^{\sharp}$. The set of all Drazin invertible (respectively group invertible) elements of $\mathcal{A}$ will be denoted by $\mathcal{A}^{\mathcal{D}}$ (respectively $\left.A^{\sharp}\right)$; see $[9,[16,28]$.

Recall that $a \in \mathcal{A}$ is said to be generalized Drazin or Koliha-Drazin invertible, if there exists $b \in A$ such that

$$
b a b=b, \quad a b=b a, \quad a b a=a+w,
$$

where $w \in \mathcal{A}^{\text {qnil }}$ (the set of all quasinilpotent elements of $\mathcal{A}$ ). The KolihaDrazin inverse is unique, if it exists, and it will be denoted by $a^{D}$; see [17, 19, 26, 27].

Note that if $a \in \mathcal{A}$ is Drazin invertible, then $a$ is generalized Drazin invertible. In fact, if $k=$ ind $(a)$, then $(a b a-a)^{k}=0$. In addition, according to [19, Proposition 1.5] (see also [17, Theorem 4.2]), necessary and sufficient for $a \in \mathcal{A}$ to be Koliha-Drazin invertible but not invertible is that $0 \in$ iso $\sigma(a)$. The set of all Koliha-Drazin invertible elements of $\mathcal{A}$ will be denoted by $\mathcal{A}^{\mathcal{K} \mathcal{D}}$.

Recall that given a Banach algebra $\mathcal{A}$, a subset $\mathcal{R} \subset \mathcal{A}$ is said to be a regularity, if the following two conditions are satisfied (see for example [18, 24]):

(i) Given $a \in \mathcal{A}$ and $n \in \mathbb{N}, a \in \mathcal{R}$ if and only if $a^{n} \in \mathcal{R}$.

(ii) If $a, b, c, d \in \mathcal{A}$ are mutually commuting elements satisfying $a c+b d=$ 1 , then necessary and sufficient for $a b \in \mathcal{R}$ is that $a, b \in \mathcal{R}$. 
Given a regularity $\mathcal{R} \subset \mathcal{A}$, it is possible to define the spectrum of $a \in \mathcal{A}$ corresponding to $\mathcal{R}$ as $\sigma_{\mathcal{R}}(a)=\{\lambda \in \mathbb{C}: a-\lambda \notin \mathcal{R}\}(a \in \mathcal{A})$. This spectrum satisfies the spectral mapping theorem for every $a \in \mathcal{A}$ and every analytic function defined on a neighbourhood of $\sigma(a)$ which is non-constant on each component of its domain of definition; see [18, Theorem 1.4]. In particular, according to [4, Theorem 2.3] and [19, Theorem 1.2], the sets $\mathcal{A}^{\mathcal{D}}=\{a \in \mathcal{A}: a$ is Drazin invertible $\}$ and $\mathcal{A}^{\mathcal{K D}}=\{a \in$ $\mathcal{A}: a$ is Koliha-Drazin invertible $\}$ are regularities, respectively. The corresponding Drazin and Koliha-Drazin spectra will be denoted by $\sigma_{\mathcal{D}}(a)$ and $\sigma_{\mathcal{K D}}(a)$, respectively.

Let $\mathcal{X}$ be a Banach space and denote by $\mathcal{L}(\mathcal{X})$ the algebra of all bounded and linear maps defined on and with values in $\mathcal{X}$. If $T \in \mathcal{L}(\mathcal{X})$, then $T^{-1}(0)$ and $R(T)$ will stand for the null space and the range of $T$ respectively. Note that $I \in \mathcal{L}(\mathcal{X})$ will denote the identity map of $\mathcal{X}$. In addition, $\mathcal{K}(\mathcal{X}) \subset \mathcal{L}(\mathcal{X})$ will stand for the closed ideal of compact operators. Consider $\mathcal{C}(\mathcal{X})$ the Calkin algebra over $\mathcal{X}$, i.e., the quotient algebra $\mathcal{C}(\mathcal{X})=\mathcal{L}(\mathcal{X}) / \mathcal{K}(\mathcal{X})$. Recall that $\mathcal{C}(\mathcal{X})$ is itself a Banach algebra with the quotient norm. Let

$$
\pi: \mathcal{L}(\mathcal{X}) \rightarrow \mathcal{C}(\mathcal{X})
$$

denote the quotient map.

Recall that $T \in \mathcal{L}(\mathcal{X})$ is said to be a Fredholm operator, if $T^{-1}(0)$ and $\mathcal{X} / R(T)$ are finite dimensional. Denote by $\Phi(\mathcal{X})$ the set of all Fredholm operators defined on $\mathcal{X}$. It is well known that $\Phi(\mathcal{X})$ is a multiplicative open semigroup in $\mathcal{L}(\mathcal{X})$ and that

$$
\Phi(\mathcal{X})=\pi^{-1}\left(\mathcal{C}(\mathcal{X})^{-1}\right) .
$$

Atkinson's theorem motivated the Fredholm theory relative to homomorphism between two Banach algebras as well as the introduction of Browder and Weyl elements relative to a such homomorphism. This theory was introduced in [11]. Next follow the main notions.

Definition 2.1. Let $\mathcal{A}$ and $\mathcal{B}$ be two unital Banach algebras and consider a (not necessarily continuous) homomorphism $\mathcal{T}: \mathcal{A} \rightarrow \mathcal{B}$. An element $a \in \mathcal{A}$ will be said to be

(i) Fredholm, if $\mathcal{T}(a)$ is invertible in $\mathcal{B}$;

(ii) Weyl, if there exist $b, c \in \mathcal{A}, b \in \mathcal{A}^{-1}$ and $c \in \mathcal{T}^{-1}(0)$, such that $a=b+c$;

(iii) Browder, if there exist $b, c \in \mathcal{A}, b \in \mathcal{A}^{-1}, c \in \mathcal{T}^{-1}(0)$ and $b c=c b$, such that $a=b+c$.

It is worth noting that the definitions of Weyl and Browder elements are also motivated from the Banach space operator case (see [11, p.431]). The sets of Fredholm, Weyl and Browder elements relative to the homomorphism $\mathcal{T}: \mathcal{A} \rightarrow \mathcal{B}$ will be denoted by $\mathcal{F}_{\mathcal{T}}(\mathcal{A}), \mathcal{W}_{\mathcal{T}}(\mathcal{A})$ and $\mathcal{B}_{\mathcal{T}}(\mathcal{A})$, respectively. Naturally, these sets lead to the introduction of the corresponding spectra.

Definition 2.2. Let $\mathcal{A}$ and $\mathcal{B}$ be two unital Banach algebras and consider a (not necessarily continuous) homomorphism $\mathcal{T}: \mathcal{A} \rightarrow \mathcal{B}$. Given $a \in \mathcal{A}$, 
the Fredholm spectrum, the Weyl spectrum and the Browder spectrum of $a$ relative to the homomorphism $\mathcal{T}: \mathcal{A} \rightarrow \mathcal{B}$ is

(i) $\sigma_{\mathcal{F}_{\mathcal{T}}}(a)=\left\{\lambda \in \mathbb{C}: a-\lambda \notin \mathcal{F}_{\mathcal{T}}(\mathcal{A})\right\}=\sigma(\mathcal{T}(a))$,

(ii) $\sigma_{\mathcal{W}_{\mathcal{T}}}(a)=\left\{\lambda \in \mathbb{C}: a-\lambda \notin \mathcal{W}_{\mathcal{T}}(\mathcal{A})\right\}$

(iii) $\sigma_{\mathcal{B}_{\mathcal{T}}}(a)=\left\{\lambda \in \mathbb{C}: a-\lambda \notin \mathcal{B}_{\mathcal{T}}(\mathcal{A})\right\}$,

respectively.

It is clear that $\mathcal{B}_{\mathcal{T}}(\mathcal{A}) \subset \mathcal{W}_{\mathcal{T}}(\mathcal{A}) \subset \mathcal{F}_{\mathcal{T}}(\mathcal{A})$ and that $\sigma_{\mathcal{F}_{\mathcal{T}}}(a) \subset \sigma_{\mathcal{W}_{\mathcal{T}}}(a) \subset$ $\sigma_{\mathcal{B}_{\mathcal{T}}}(a) \subset \sigma(a)$. Also it is known that the sets $\sigma_{\mathcal{F}_{\mathcal{T}}}(a), \sigma_{\mathcal{W}_{\mathcal{T}}}(a)$ and $\sigma_{\mathcal{B}_{\mathcal{T}}}(a)$ are non-empty and compact. To learn the main properties of these objects, see for example [11, 12, 13, 14, 20, 10, 21, 8, 15, 1, 30, 22, 31, 32.

Recall that an operator $T \in \mathcal{L}(\mathcal{X})$ is said to be $B$-Fredholm, if there is $n \in \mathbb{N}$ such that $R\left(T^{n}\right)$ is closed and $\left.T\right|_{R\left(T^{n}\right)} \in \mathcal{L}\left(R\left(T^{n}\right)\right)$ is Fredholm, see [2, 23. Denote by $\mathcal{B F}(\mathcal{X})$ the class of B-Fredholm operators defined on the Banach space $\mathcal{X}$. According to [4, Theorem 3.4], $T \in \mathcal{B F}(\mathcal{X})$ if and only if $\tilde{\pi}(T) \in(\mathcal{L}(\mathcal{X}) / \mathcal{F}(\mathcal{X}))^{\mathcal{D}}$, where $\mathcal{F}(\mathcal{X})$ denotes the ideal of finite rank operators defined on $\mathcal{X}$ and $\tilde{\pi}: \mathcal{L}(\mathcal{X}) \rightarrow \mathcal{L}(\mathcal{X}) / \mathcal{F}(\mathcal{X})$ is the quotient homomorphism. In addition, given a Hilbert space $\mathcal{H}$, according to [7, Theorem 3.12(ii)], $\pi(\mathcal{B} \mathcal{F}(\mathcal{H}))=\mathcal{C}(\mathcal{H})^{D}$. Moreover, B-Weyl operators were introduced in [3] and according to [3, Corollary 4.4], $T \in \mathcal{L}(\mathcal{X})$ is a B-Weyl if and only if $T=S+F$, where $S \in \mathcal{L}(\mathcal{X})^{\mathcal{D}}$ and $F \in \mathcal{F}(\mathcal{X})$.

On the other hand, the classes of Riesz-Fredholm and power compactFredholm operators on the Banach space $\mathcal{X}$ were introduced in [7] and they will be denoted by $\mathcal{R} \mathcal{F}(\mathcal{X})$ and $\mathcal{P} \mathcal{K} \mathcal{F}(\mathcal{X})$, respectively. According to [7, Theorem 3.11], $\pi(\mathcal{R} \mathcal{F}(\mathcal{X}))=\mathcal{C}(\mathcal{X})^{\mathcal{K D}}$ and $\pi(\mathcal{P K \mathcal { F }}(\mathcal{X}))=\mathcal{C}(\mathcal{X})^{\mathcal{D}}$.

These observations led to the following definition.

Definition 2.3. Let $\mathcal{A}$ and $\mathcal{B}$ be two unital Banach algebras and consider a (not necessarily continuous) homomorphism $\mathcal{T}: \mathcal{A} \rightarrow \mathcal{B}$. An element $a \in \mathcal{A}$ is said to be

(i) B-Fredholm (respectively $B$-Fredholm of degree $k$ ), if $\mathcal{T}(a) \in \mathcal{B}^{\mathcal{D}}$ (respectively $\mathcal{T}(a) \in \mathcal{B}^{\mathcal{D}}$ and ind $\left.T(a)=k\right)$;

(ii) $B$-Weyl (respectively $B$-Weyl of degree $k$ ), if there exist $b, c \in \mathcal{A}, b \in \mathcal{A}^{\mathcal{D}}$ (respectively $b \in \mathcal{A}^{\mathcal{D}}$ and ind $b=k$ ) and $c \in \mathcal{T}^{-1}(0)$, such that $a=b+c$;

(iii) $B$-Browder $(B$-Browder of degree $k$ ) if there exist $b, c \in \mathcal{A}, b c=c b$, $b \in \mathcal{A}^{\mathcal{D}}$ (respectively $b \in \mathcal{A}^{\mathcal{D}}$ and ind $b=k$ ) and $c \in \mathcal{T}^{-1}(0)$, such that $a=b+c$;

(iv) generalized $B$-Fredholm, if $\mathcal{T}(a) \in \mathcal{B}^{\mathcal{K D}}$;

(v) generalized $B$-Weyl, if there exist $b, c \in \mathcal{A}, b \in \mathcal{A}^{\mathcal{K D}}$ and $c \in \mathcal{T}^{-1}(0)$, such that $a=b+c$;

(vi) generalized $B$-Browder, if there exist $b, c \in \mathcal{A}, b c=c b, b \in \mathcal{A}^{\mathcal{K D}}$ and $c \in \mathcal{T}^{-1}(0)$, such that $a=b+c$.

The set of B-Fredholm (respectively B-Fredholm of degree $k$, B-Weyl, B-Weyl of degree $k$, B-Browder, B-Browder of degree $k$, generalized BFredholm, generalized B-Weyl and generalized B-Browder) elements of the unital Banach algebra $\mathcal{A}$ relative to the homomorphism $\mathcal{T}: \mathcal{A} \rightarrow \mathcal{B}$ will be denoted by $\mathcal{B F}_{\mathcal{T}}(\mathcal{A})$ (respectively $\mathcal{B F}_{\mathcal{T}}^{(k)}(\mathcal{A}), \mathcal{B W}_{\mathcal{T}}(\mathcal{A}), \mathcal{B W}_{\mathcal{T}}^{(k)}(\mathcal{A}), \mathcal{B B}_{\mathcal{T}}(A)$, 
$\mathcal{B B}_{\mathcal{T}}^{(k)}(\mathcal{A}), \mathcal{G B F}_{\mathcal{T}}(\mathcal{A}), \mathcal{G B}_{\mathcal{T}}(\mathcal{A})$ and $\left.\mathcal{G B B}_{\mathcal{T}}(\mathcal{A})\right)$. Next the corresponding spectra will be introduced.

Definition 2.4. Let $\mathcal{A}$ and $\mathcal{B}$ be two unital Banach algebras and consider a (not necessarily continuous) homomorphism $\mathcal{T}: \mathcal{A} \rightarrow \mathcal{B}$. Given $a \in \mathcal{A}$, the $B$-Fredholm spectrum, the $B$-Weyl spectrum, the B-Browder spectrum, the generalized B-Fredholm spectrum, the generalized $B$-Weyl spectrum and the generalized $B$-Browder spectrum of $a$ relative to the homomorphism $\mathcal{T}: \mathcal{A} \rightarrow \mathcal{B}$ is

(i) $\sigma_{\mathcal{B F}_{\mathcal{T}}}(a)=\left\{\lambda \in \mathbb{C}: a-\lambda \notin \mathcal{B F}_{\mathcal{T}}(\mathcal{A})\right\}=\sigma_{\mathcal{D}}(\mathcal{T}(a))$

(ii) $\sigma_{\mathcal{B} \mathcal{W}_{\mathcal{T}}}(a)=\left\{\lambda \in \mathbb{C}: a-\lambda \notin \mathcal{B} \mathcal{W}_{\mathcal{T}}(\mathcal{A})\right\}$,

(iii) $\sigma_{\mathcal{B B}_{\mathcal{T}}}(a)=\left\{\lambda \in \mathbb{C}: a-\lambda \notin \mathcal{B B}_{\mathcal{T}}(\mathcal{A})\right\}$,

(iv) $\sigma_{\mathcal{G B F}_{\mathcal{T}}}(a)=\left\{\lambda \in \mathbb{C}: a-\lambda \notin \mathcal{G B \mathcal { F }}_{\mathcal{T}}(\mathcal{A})\right\}=\sigma_{\mathcal{K D}}(\mathcal{T}(a))$,

(v) $\sigma_{\mathcal{G B W}}(a)=\left\{\lambda \in \mathbb{C}: a-\lambda \notin \mathcal{G B} \mathcal{W}_{\mathcal{T}}(\mathcal{A})\right\}$

(vi) $\sigma_{\mathcal{G B B}}(a)=\left\{\lambda \in \mathbb{C}: a-\lambda \notin \mathcal{G B B}_{\mathcal{T}}(\mathcal{A})\right\}$,

respectively.

Remark 2.5. Let $\mathcal{A}$ and $\mathcal{B}$ be two unital Banach algebras and consider a (not necessarily continuous) homomorphism $\mathcal{T}: \mathcal{A} \rightarrow \mathcal{B}$ and $a \in A$. Then, it is not difficult to prove the following statements:

(i) $\mathcal{F}_{\mathcal{T}}(\mathcal{A}) \subseteq \mathcal{B F}_{\mathcal{T}}(\mathcal{A}) \subseteq \mathcal{G B F}_{\mathcal{T}}(\mathcal{A})$

(ii) $\mathcal{B F}_{\mathcal{T}}(\mathcal{A})+\mathcal{T}^{-1}(0)=\mathcal{B F}_{\mathcal{T}}(\mathcal{A})$.

(iii) $\mathcal{W}_{\mathcal{T}}(\mathcal{A}) \subseteq \mathcal{B W}_{\mathcal{T}}(\mathcal{A})=\mathcal{A}^{\mathcal{D}}+\mathcal{T}^{-1}(0) \subseteq \mathcal{A}^{\mathcal{K D}}+\mathcal{T}^{-1}(0)=\mathcal{G B} \mathcal{W}_{\mathcal{T}}(\mathcal{A})$ and $\mathcal{B}_{\mathcal{T}}(\mathcal{A}) \subseteq \mathcal{B B}_{\mathcal{T}}(\mathcal{A}) \subseteq \mathcal{G B B}_{\mathcal{T}}(\mathcal{A})$

(iv) $\mathcal{A}^{\mathcal{D}} \subseteq \mathcal{B B}_{\mathcal{T}}(\mathcal{A}) \subseteq \mathcal{B W}_{\mathcal{T}}(\mathcal{A}) \subseteq \mathcal{B F}_{\mathcal{T}}(\mathcal{A})$ and $\mathcal{A}^{\mathcal{K D}} \subseteq \mathcal{G B B}_{\mathcal{T}}(\mathcal{A}) \subseteq$ $\mathcal{G B W}_{\mathcal{T}}(\mathcal{A}) \subseteq \mathcal{G B F}_{\mathcal{T}}(\mathcal{A})$

(v) $\mathcal{G B F}_{\mathcal{T}}(\mathcal{A})+\mathcal{T}^{-1}(0)=\mathcal{G B F}_{\mathcal{T}}(\mathcal{A})$

(vi) $\mathcal{A}^{\mathcal{D}} \subseteq \mathcal{A}^{\mathcal{K D}} \subseteq \mathcal{G B F}_{\mathcal{T}}(\mathcal{A})$

(vii) $\sigma_{\mathcal{G B F}_{\mathcal{T}}}(a) \subseteq \sigma_{\mathcal{B F}_{\mathcal{T}}}(a) \subseteq \sigma_{\mathcal{F}_{\mathcal{T}}}(a)$.

(viii) $\sigma_{\mathcal{G B} \mathcal{W}_{\mathcal{T}}}(a) \subseteq \sigma_{\mathcal{B W}_{\mathcal{T}}}(a) \subseteq \sigma_{\mathcal{W}_{\mathcal{T}}}(a)$ and $\sigma_{\mathcal{G B B}_{\mathcal{T}}}(a) \subseteq \sigma_{\mathcal{B B}}(a) \subseteq \sigma_{\mathcal{B}_{\mathcal{T}}}(a)$.

(ix) $\sigma_{\mathcal{B} \mathcal{F}_{\mathcal{T}}}(a) \subseteq \sigma_{\mathcal{B W}_{\mathcal{T}}}(a) \subseteq \sigma_{\mathcal{B B}_{\mathcal{T}}}(a) \subseteq \sigma_{\mathcal{D}}(a)$ and $\sigma_{\mathcal{G B \mathcal { F }}}(a) \subseteq \sigma_{\mathcal{G} \mathcal{B W}_{\mathcal{T}}}(a) \subseteq$ $\sigma_{\mathcal{G B B}}(a) \subseteq \sigma_{\mathcal{K} \mathcal{D}}(a)$

$(\mathrm{x}) \sigma_{\mathcal{G B F}}(a) \subseteq \sigma_{\mathcal{K} \mathcal{D}}(a) \subseteq \sigma_{\mathcal{D}}(a)$.

Finally, given $S \subset \mathcal{A}$, $\operatorname{Poly}^{-1}(S)=\{a \in \mathcal{A}: \exists p \in \mathbb{C}[X], p \neq 0$ and $p(a) \in$ $S\}$, where $\mathbb{C}[X]$ is the algebra of complex polynomials. In particular, Poly ${ }^{-1}(\{$ $0\}$ ) is the set of algebraic elements of $\mathcal{A}$.

\section{Generalized B-Fredholm elements}

In this section, the basic properties of the objects studied in this work will be considered. To this end, however, first it is necessary to recall some facts.

Remark 3.1. Let $\mathcal{A}$ be a unital Banach algebra and consider $a \in \mathcal{A}$. Recall that according to [5, Theorem 12(iv)], $\sigma_{\mathcal{D}}(a)=I(a) \cup$ acc $\sigma(a)$, where $I(a)=$ iso $\sigma(a) \backslash \Pi(a)$ and $\Pi(a) \subseteq$ iso $\sigma(a)$ denotes the set of poles of $a \in \mathcal{A}$ ([5, Remark 10]). In particular $\operatorname{acc} \sigma_{\mathcal{D}}(a)=\operatorname{acc} \sigma(a)=\sigma_{\mathcal{K D}}(a)$ ([5, Theorem 
12(iii)] and [19, Proposition 1.5(i)]). The following statements can be easily deduced from this fact.

(i) Let $b \in \mathcal{A}$. Since $\sigma_{\mathcal{D}}(a b)=\sigma_{\mathcal{D}}(b a)\left(\left[6\right.\right.$, Theorem 2.3]), $\sigma_{\mathcal{K D}}(a b)=\sigma_{\mathcal{K D}}(b a)$.

(ii) According to [6, Theorem 2.2], necessary and sufficient for $\sigma_{\mathcal{D}}(a)$ to be countable is that $\sigma(a)$ is countable. As a result, $\sigma_{\mathcal{K D}}(a)$ is countable if and only if $\sigma_{\mathcal{D}}(a)$ is countable if and only if $\sigma(a)$ is countable.

In the following theorem the main properties of the (generalized) BFredholm spectrum will be studied.

Theorem 3.2. Let $\mathcal{A}$ and $\mathcal{B}$ be two unital Banach algebras and consider a (not necessarily continuous) homomorphism $\mathcal{T}: \mathcal{A} \rightarrow \mathcal{B}$. If $a \in \mathcal{A}$, then the following statements hold.

(i) $\mathcal{B F}_{\mathcal{T}}(\mathcal{A})$ and $\mathcal{G B F}_{\mathcal{T}}(\mathcal{A})$ are regularities.

(ii) If $f: U \rightarrow \mathbb{C}$ is an analytic function defined on a neighbourhood of $\sigma(a)$ which is non-constant on each component of its domain of definition, then

$$
\sigma_{\mathcal{B F}_{\mathcal{T}}}(f(a))=f\left(\sigma_{\mathcal{B F}_{\mathcal{T}}}(a)\right) \text {, and } \sigma_{\mathcal{G B F}_{\mathcal{T}}}(f(a))=f\left(\sigma_{\mathcal{G B F}_{\mathcal{T}}}(a)\right) \text {. }
$$

(iii) $\sigma_{\mathcal{B F}_{\mathcal{T}}}(a)$ and $\sigma_{\mathcal{G B F}_{\mathcal{T}}}($ a) are closed.

(iv) $\sigma_{\mathcal{B F}_{\mathcal{T}}}(a)=\emptyset$ if and only if $a \in \operatorname{Poly}^{-1}\left(\mathcal{T}^{-1}(0)\right)$, equivalently, $\mathcal{T}(a) \in$ Poly $^{-1}(0)$.

(v) $\sigma_{\mathcal{G B F}_{\mathcal{T}}}(a)=\emptyset$ if and only if acc $\sigma_{\mathcal{F}_{\mathcal{T}}}(a)=\emptyset$.

(vi) Given $a_{1}$ and $a_{2} \in \mathcal{A}, \sigma_{\mathcal{B F}_{\mathcal{T}}}\left(a_{1} a_{2}\right)=\sigma_{\mathcal{B F}_{\mathcal{T}}}\left(a_{2} a_{1}\right)$ and $\sigma_{\mathcal{G B F}_{\mathcal{T}}}\left(a_{1} a_{2}\right)=$ $\sigma_{\mathcal{G B F}_{\mathcal{T}}}\left(a_{2} a_{1}\right)$.

(vii) $\sigma_{\mathcal{B F}_{\mathcal{T}}}(a)$ is countable if and only if $\sigma_{\mathcal{G B F}_{\mathcal{T}}}(a)$ is countable if and only if $\sigma_{\mathcal{F}_{\mathcal{T}}}(a)$ is countable.

Proof. (i). According to [4, Theorem 2.3] and [19, Theorem 1.2], $\mathcal{A}^{\mathcal{D}}$ and $\mathcal{A}^{\mathcal{K D}}$ are regularities, respectively. Since $\mathcal{T}: \mathcal{A} \rightarrow \mathcal{B}$ is an algebra homomorphism, $\mathcal{B F}_{\mathcal{T}}(\mathcal{A})$ and $\mathcal{G B F}_{\mathcal{T}}(\mathcal{A})$ are regularities.

(ii). Apply [18, Theorem 1.4] to $\mathcal{B F}_{\mathcal{T}}(\mathcal{A})$ and $\mathcal{G B F}_{\mathcal{T}}(\mathcal{A})$.

(iii). Recall that $\sigma_{\mathcal{B} \mathcal{F}_{\mathcal{T}}}(a)=\sigma_{\mathcal{D}}(\mathcal{T}(a))$ and $\sigma_{\mathcal{G B F}}(a)=\sigma_{\mathcal{K} \mathcal{D}}(\mathcal{T}(a))$. Then, apply [4, Proposition 2.5] and [19, Proposition 1.5(ii)].

(iv). Since $\sigma_{\mathcal{B F}_{\mathcal{T}}}(a)=\sigma_{\mathcal{D}}(\mathcal{T}(a))$, this statement can be deduced from 6 , Theorem 2.1].

(v). Recall that, according to [19, Proposition 1.5(i)], $\sigma_{\mathcal{G B F}_{\mathcal{T}}}(a)=\sigma_{\mathcal{K D}}(\mathcal{T}(a))=$ $\operatorname{acc} \sigma(\mathcal{T}(a))=\operatorname{acc} \sigma_{\mathcal{F}_{\mathcal{T}}}(a)$.

(vi). Apply [6, Theorem 2.3] and Remark 3.1(i).

(vii). Apply [6, Theorem 2.2] and Remark 3.1(ii).

Next, (generalized) B-Fredholm elements will be characterized. To this end, however, some notions need to be recalled.

Let $\mathcal{A}$ and $\mathcal{B}$ be two unital Banach algebras and consider a (not necessarily continuous) homomorphism $\mathcal{T}: \mathcal{A} \rightarrow \mathcal{B}$. The kernel and the range of the homomorphism $\mathcal{T}$ will be denoted by $\mathcal{T}^{-1}(0)$ and $R(\mathcal{T})$, respectively. Let $\mathcal{R}_{\mathcal{T}}(\mathcal{A})=\left\{a \in \mathcal{A}: \mathcal{T}(a) \in \mathcal{B}^{\text {qnil }}\right\}$ be the set of Riesz elements of $\mathcal{A}$ relative to the homomorphism $\mathcal{T}$ and $\mathcal{N}_{\mathcal{T}}(\mathcal{A})=\{a \in \mathcal{A}$ : there exists $k \in$ $\mathbb{N}$ such that $\left.a^{k} \in \mathcal{T}^{-1}(0)\right\}=\left\{a \in \mathcal{A}: \mathcal{T}(a) \in \mathcal{B}^{\text {nil }}\right\}$ be the set of $\mathcal{T}$-nilpotent elements of $\mathcal{A}$; see [7, 31, 32]. Clearly, $\mathcal{N}_{\mathcal{T}}(\mathcal{A}) \subseteq \mathcal{R}_{\mathcal{T}}(\mathcal{A})$. 
On the other hand, the homomorphism $\mathcal{T}: \mathcal{A} \rightarrow \mathcal{B}$ will be said to have the lifting property, if given $q \in \mathcal{B}^{\bullet}$, there is $p \in \mathcal{A}^{\bullet}$ such that $\mathcal{T}(p)=q$, i.e., $T\left(\mathcal{A}^{\bullet}\right)=\mathcal{B}^{\bullet}$, which is equivalent to the conjunction of the following two conditions: $\mathcal{T}^{-1}\left(\mathcal{B}^{\bullet}\right)=\mathcal{A}^{\bullet}+\mathcal{T}^{-1}(0)$ and $\mathcal{B}^{\bullet} \subset R(\mathcal{T})$. This property does not in general hold. Some examples that satisfy the lifting property (among others the radical of a Banach algebra or von Neumann algebras) were considered in [7, Remark 3.4]. In particular, if $\mathcal{B}^{\bullet} \subset R(\mathcal{T})$ and $\mathcal{T}$ has the Riesz property, i.e., if for every $z \in \mathcal{T}^{-1}(0), \sigma(z)$ is either finite or is a sequence converging to 0 , then $\mathcal{T}$ has the lifting property, see [25, Theorem 1] and [8, Lemma 2]. Therefore, if $\mathcal{T}: \mathcal{A} \rightarrow \mathcal{B}$ is surjective and has the Riesz property, then $\mathcal{T}$ has the lifting property.

Theorem 3.3. Let $\mathcal{A}$ and $\mathcal{B}$ be two unital Banach algebras and consider a (not necessarily continuous) homomorphism $\mathcal{T}: \mathcal{A} \rightarrow \mathcal{B}$. Suppose that $\mathcal{T}$ has the lifting property. Then, the following statements hold.

(i) Necessary and sufficient for $a \in \mathcal{G B F}_{\mathcal{T}}(\mathcal{A})$ is that there exists $p \in \mathcal{A}^{\bullet}$ such that $a+p \in \mathcal{F}_{\mathcal{T}}(\mathcal{A})$, pa $(1-p)$ and $(1-p)$ ap $\in \mathcal{T}^{-1}(0)$ and pap $\in \mathcal{R}_{\mathcal{T}}(\mathcal{A})$. (ii) Necessary and sufficient for $a \in \mathcal{B F}_{\mathcal{T}}(\mathcal{A})$ is that there exists $p \in \mathcal{A}^{\bullet}$ such that $a+p \in \mathcal{F}_{\mathcal{T}}(\mathcal{A}), p a(1-p)$ and $(1-p)$ ap $\in \mathcal{T}^{-1}(0)$ and pap $\in \mathcal{N}_{\mathcal{T}}(\mathcal{A})$.

Proof. (i). If $a \in \mathcal{G B F}_{\mathcal{T}}(\mathcal{A})$, then $\mathcal{T}(a) \in \mathcal{B}^{\mathcal{K D}}$. In particular, according to [17, Theorem 4.2], there is $q \in \mathcal{B}^{\bullet}$ such that $q \mathcal{T}(a)=\mathcal{T}(a) q, \mathcal{T}(a)+q \in \mathcal{B}^{-1}$ and $\mathcal{T}(a) q=q \mathcal{T}(a) q \in \mathcal{B}^{\text {qnil }}$. Since $\mathcal{T}: \mathcal{A} \rightarrow \mathcal{B}$ has the lifting property, there is $p \in \mathcal{A}^{\bullet}$ such that $\mathcal{T}(p)=q$.

Now, the identity $q \mathcal{T}(a)=\mathcal{T}(a) q$ implies that $p a-a p \in \mathcal{T}^{-1}(0)$. However, multiplying by $1-p$, it is easy to prove that $p a(1-p)$ and $(1-p) a p \in$ $\mathcal{T}^{-1}(0)$. In addition, since $\mathcal{T}(a+p) \in \mathcal{B}^{-1}, a+p \in \mathcal{F}_{\mathcal{T}}(\mathcal{A})$. Finally, since $\mathcal{T}($ pap $) \in \mathcal{B}^{\text {qnil }}$, pap $\in \mathcal{R}_{\mathcal{T}}(\mathcal{A})$.

Suppose that there exists $p \in \mathcal{A}^{\bullet}$ such that $a+p \in \mathcal{F}_{\mathcal{T}}(\mathcal{A}), p a(1-p)$ and $(1-p) a p \in \mathcal{T}^{-1}(0)$ and pap $\in \mathcal{R}_{\mathcal{T}}(\mathcal{A})$. Consequently, $q=\mathcal{T}(p) \in \mathcal{B}^{\bullet}$ and $q \mathcal{T}(a)=\mathcal{T}(a) q, \mathcal{T}(a)+q \in \mathcal{B}^{-1}$ and $\mathcal{T}(a) q=q \mathcal{T}(a) q \in \mathcal{B}^{\text {qnil }}$. Thus, according to [17, Theorem 4.2], $\mathcal{T}(a) \in \mathcal{B}^{\mathcal{K D}}$, equivalently, $a \in \mathcal{G B F}_{\mathcal{T}}(\mathcal{A})$. (ii). Apply the same argument used in the proof of statement (i), using in particular [28, Proposition 1(a)] instead of [17, Theorem 4.2].

Next some basic properties of the objects introduced in Definition 2.3 will be considered.

Theorem 3.4. Let $\mathcal{A}$ and $\mathcal{B}$ be two unital Banach algebras and consider a (not necessarily continuous) homomorphism $\mathcal{T}: \mathcal{A} \rightarrow \mathcal{B}$. Then, the following statements hold.

(i) $\mathcal{T}^{-1}(0) \subseteq \mathcal{T}^{-1}\left(\mathcal{B}^{\bullet}\right) \subseteq \mathcal{B F}_{\mathcal{T}}(\mathcal{A})$.

(ii) $\mathcal{A}^{\bullet} \subseteq \mathcal{T}^{-1}\left(\mathcal{B}^{\bullet}\right) \subseteq \mathcal{B F}_{\mathcal{T}}(\mathcal{A})$.

(iii) $\mathcal{F}_{\mathcal{T}}(\mathcal{A})$ is a proper subset of $\mathcal{B F}_{\mathcal{T}}(\mathcal{A})$.

(iv) $\mathcal{W}_{\mathcal{T}}(\mathcal{A})$ is a proper subset of $\mathcal{B} \mathcal{W}_{\mathcal{T}}(\mathcal{A})$.

(v) $\mathcal{B}_{\mathcal{T}}(\mathcal{A})$ is a proper subset of $\mathcal{B B}_{\mathcal{T}}(\mathcal{A})$.

(vi) $\mathcal{A}^{\bullet} \backslash \mathcal{T}^{-1}(1) \subseteq \mathcal{B F}_{\mathcal{T}}(\mathcal{A}) \backslash \mathcal{F}_{\mathcal{T}}(\mathcal{A})$.

(vii) If $a, b \in \mathcal{B F}_{\mathcal{T}}(\mathcal{A})$ are such that $a b-b a \in \mathcal{T}^{-1}(0)$, then $a b \in \mathcal{B F}_{\mathcal{T}}(\mathcal{A})$.

(viii) If $a \in \mathcal{B} \mathcal{W}_{\mathcal{T}}(\mathcal{A})$, then $a^{n} \in \mathcal{B} \mathcal{W}_{\mathcal{T}}(\mathcal{A})$ for every $n \in \mathbb{N}$. 
(ix) If $a \in \mathcal{B B}_{\mathcal{T}}(\mathcal{A})$, then $a^{n} \in \mathcal{B B}_{\mathcal{T}}(\mathcal{A})$ for every $n \in \mathbb{N}$.

$(\mathrm{x}) \mathcal{B} \mathcal{W}_{\mathcal{T}}(\mathcal{A}) \backslash \mathcal{W}_{\mathcal{T}}(\mathcal{A}) \subseteq \mathcal{B F}_{\mathcal{T}}(\mathcal{A}) \backslash \mathcal{F}_{\mathcal{T}}(\mathcal{A})$

(xi) $\mathcal{B B}_{\mathcal{T}}(\mathcal{A}) \backslash \mathcal{B}_{\mathcal{T}}(\mathcal{A}) \subseteq \mathcal{B F}_{\mathcal{T}}(\mathcal{A}) \backslash \mathcal{F}_{\mathcal{T}}(\mathcal{A})$

(xii) $\mathcal{A}^{\mathcal{K D}} \cap \mathcal{B F}_{\mathcal{T}}^{(1)}(\mathcal{A}) \subseteq \mathcal{B B}_{\mathcal{T}}^{(1)}(\mathcal{A})$.

(xiii) $\sigma_{\mathcal{B} \mathcal{W}_{\mathcal{T}}}(a)=\bigcap_{c \in \mathcal{T}^{-1}(0)} \sigma_{\mathcal{D}}(a+c)(a \in \mathcal{A})$.

(xiv) $\sigma_{\mathcal{B B}}(a)=\bigcap_{c \in \mathcal{T}^{-1}(0), a c=c a} \sigma_{\mathcal{D}}(a+c)(a \in \mathcal{A})$.

$(\mathrm{xv})$ The sets $\sigma_{\mathcal{B} \mathcal{W}_{\mathcal{T}}}(a)$ and $\sigma_{\mathcal{B B}}(a)$ are closed $(a \in \mathcal{A})$.

Proof. (i). This statement can be easily derived from the inclusions

$$
\{0\} \subseteq \mathcal{B}^{\bullet} \subseteq \mathcal{B}^{\mathcal{D}}
$$

(ii). Clearly, $\mathcal{T}\left(\mathcal{A}^{\bullet}\right) \subseteq \mathcal{B}^{\bullet}$ and $\mathcal{B}^{\bullet} \subseteq \mathcal{B}^{\mathcal{D}}$.

(iii). Since $\{0\} \cap \mathcal{B}^{-1}=\emptyset$, then $\mathcal{T}^{-1}(0) \cap \mathcal{F}_{\mathcal{T}}(\mathcal{A})=\mathcal{T}^{-1}(0) \cap T^{-1}\left(\mathcal{B}^{-1}\right)=\emptyset$.

Consequently, $\mathcal{T}^{-1}(0) \subseteq \mathcal{B F}_{\mathcal{T}}(\mathcal{A}) \backslash \mathcal{F}_{\mathcal{T}}(\mathcal{A})$

(iv). Clearly, $\mathcal{T}^{-1}(0) \subseteq \mathcal{B} \mathcal{W}_{\mathcal{T}}(\mathcal{A})$. In addition, according to the proof of statement (iii), $\mathcal{T}^{-1}(0) \cap \mathcal{W}_{\mathcal{T}}(\mathcal{A}) \subseteq \mathcal{T}^{-1}(0) \cap \mathcal{F}_{\mathcal{T}}(\mathcal{A})=\emptyset$. Therefore, $\mathcal{T}^{-1}(0) \subseteq$ $\mathcal{B} \mathcal{W}_{\mathcal{T}}(\mathcal{A}) \backslash \mathcal{W}_{\mathcal{T}}(\mathcal{A})$

(v). It is clear that $\mathcal{T}^{-1}(0) \subseteq \mathcal{B B}_{\mathcal{T}}(\mathcal{A}) \backslash \mathcal{B}_{\mathcal{T}}(\mathcal{A})$.

(vi). Note that $\mathcal{A}^{\bullet} \backslash \mathcal{T}^{-1}(1) \subseteq \mathcal{A}^{\bullet} \subseteq \mathcal{B F}_{\mathcal{T}}(\mathcal{A})$. In addition, if $a \in \mathcal{A}^{\bullet} \backslash \mathcal{T}^{-1}(1)$, then $\mathcal{T}(a) \in \mathcal{B}^{\bullet} \backslash \mathcal{B}^{-1}$. In particular, $a \notin \mathcal{F}_{\mathcal{T}}(\mathcal{A})$.

(vii). Apply [4, Proposition 2.6].

(viii). Let $a \in \mathcal{B} \mathcal{W}_{\mathcal{T}}(\mathcal{A})$. Then $a=b+c$, where $b \in \mathcal{A}^{\mathcal{D}}$ and $c \in \mathcal{T}^{-1}(0)$. It will be proved that $a^{n}=b^{n}+x_{n}$, where $x_{n} \in \mathcal{T}^{-1}(0)$, for every $n \in \mathbb{N}$. In fact, for $n=1$ it is obvious. Suppose that this statement is true for $k \in \mathbb{N}$. Then,

$$
a^{k+1}=a^{k} a=\left(b^{k}+x_{k}\right)(b+c)=b^{k+1}+\left(b^{k} c+x_{k} b+x_{k} c\right) .
$$

Clearly, $b^{k} c+x_{k} b+x_{k} c \in \mathcal{T}^{-1}(0)$. As a result, since $b^{n} \in \mathcal{A}^{\mathcal{D}}, a^{n} \in$ $\mathcal{B} \mathcal{W}_{\mathcal{T}}(\mathcal{A}), n \in \mathbb{N}$.

(ix). Let $a \in \mathcal{B B}_{\mathcal{T}}(\mathcal{A})$. In particular, there are $b \in \mathcal{A}^{\mathcal{D}}$ and $c \in \mathcal{T}^{-1}(0)$ such that $a=b+c$ and $b c=c b$. As a result, for every $n \in \mathbb{N}$

$$
a^{n}=(b+c)^{n}=\sum_{k=0}^{n}\left(\begin{array}{l}
n \\
k
\end{array}\right) b^{n-k} c^{k}=b^{n}+\sum_{k=1}^{n}\left(\begin{array}{l}
n \\
k
\end{array}\right) b^{n-k} c^{k} .
$$

Since $b^{n} \in \mathcal{A}^{\mathcal{D}}, z=\sum_{k=1}^{n}\left(\begin{array}{l}n \\ k\end{array}\right) b^{n-k} c^{k} \in \mathcal{T}^{-1}(0)$ and $b^{n}$ commutes with $z$, $a^{n} \in \mathcal{B B}_{\mathcal{T}}(\mathcal{A})$.

(x). Clearly, $\mathcal{B W}_{\mathcal{T}}(\mathcal{A}) \backslash \mathcal{W}_{\mathcal{T}}(\mathcal{A}) \subseteq \mathcal{B} \mathcal{W}_{\mathcal{T}}(\mathcal{A}) \subseteq \mathcal{B F}_{\mathcal{T}}(\mathcal{A})$. If $a \in \mathcal{B} \mathcal{W}_{\mathcal{T}}(\mathcal{A}) \backslash$ $\mathcal{W}_{\mathcal{T}}(\mathcal{A})$, then there exist $c \in \mathcal{A}^{\mathcal{D}}$ and $d \in \mathcal{T}^{-1}(0)$ such that $a=c+d$. In addition, according to [28, Proposition 1(a)], there is $p \in \mathcal{A}^{\bullet}$ such that

$$
c p=p c, \quad c+p \in \mathcal{A}^{-1}, \quad c p \text { is nilpotent. }
$$

Note that since $a=(c+p)+(d-p)$ and $a \notin W_{\mathcal{T}}(\mathcal{A})=\mathcal{A}^{-1}+\mathcal{T}^{-1}(0)$, $p \notin \mathcal{T}^{-1}(0)$. Let $0 \neq q=\mathcal{T}(p) \in \mathcal{B}^{\bullet}$. Then, $q \mathcal{T}(c)=\mathcal{T}(c) q, \mathcal{T}(c)+q \in \mathcal{B}^{-1}$ and $\mathcal{T}(c) q$ is nilpotent. Thus, $\mathcal{T}(c)$ is Drazin invertible but not invertible $(q \neq 0)$, which implies that $c \notin \mathcal{F}_{\mathcal{T}}(\mathcal{A})$. However, since $d \in \mathcal{T}^{-1}(0), a \notin$ $\mathcal{F}_{\mathcal{T}}(\mathcal{A})$. 
(xi). Apply an argument similar to the one used in the proof of statement $(\mathrm{x})$.

(xii). Since $\mathcal{A}^{-1} \subseteq \mathcal{B B}_{\mathcal{T}}^{(1)}(\mathcal{A})$, suppose that $a \in \mathcal{A}^{\mathcal{K D}} \backslash \mathcal{A}^{-1}$. According to [17, Theorem 6.4], there is $p \in \mathcal{A}^{\bullet}$ such that $p a=a p, a+p \in \mathcal{A}^{-1}$ and $a=x+y$, where $y=a p \in \mathcal{A}^{q n i l}, x=a(1-p), x y=y x=0$ and $x \in \mathcal{A}^{\sharp}$. In addition, since $\mathcal{T}(a) \in \mathcal{B}^{\sharp}$, there is $q \in \mathcal{B}^{\bullet}$ such that $q \mathcal{T}(a)=\mathcal{T}(a) q=0$ and $\mathcal{T}(a)+q \in \mathcal{B}^{-1}([28$, Lemma 3]). Now well, if $q=0$, then by [21, Theorem 2.4] $a \in \mathcal{F}_{\mathcal{T}}(\mathcal{A}) \cap \mathcal{A}^{\mathcal{K D}} \subseteq \mathcal{B}_{\mathcal{T}}(\mathcal{A}) \subseteq \mathcal{B B}_{\mathcal{T}}^{(1)}(\mathcal{A})$. On the other hand, if $q \neq 0$, then according to [17, Theorem 3.1], $\mathcal{T}(p)=q$ and $\mathcal{T}(y)=\mathcal{T}(a) \mathcal{T}(p)=0$. Thus, $a \in \mathcal{B B}_{\mathcal{T}}^{(1)}(\mathcal{A})$.

(xiii)-(xiv). These statements can be easily deduced.

(xv). Apply statements (xiii)-(xiv).

The following theorem summarizes the basic properties of generalized BFredholm elements.

Theorem 3.5. Let $\mathcal{A}$ and $\mathcal{B}$ be two unital Banach algebras and consider a (not necessarily continuous) homomorphism $\mathcal{T}: \mathcal{A} \rightarrow \mathcal{B}$. Then, the following statements hold.

(i) $\mathcal{T}^{-1}\left(\mathcal{B}^{\text {qnil }} \backslash \mathcal{B}^{\text {nil }}\right) \subseteq \mathcal{G B F}_{\mathcal{T}}(\mathcal{A}) \backslash \mathcal{B F}_{\mathcal{T}}(\mathcal{A})$

(ii) If $a, b \in \mathcal{G B F}_{\mathcal{T}}(\mathcal{A})$ are such that $a b-b a \in \mathcal{T}^{-1}(0)$, then $a b \in \mathcal{G B F}_{\mathcal{T}}(\mathcal{A})$.

(iii) If $a \in \mathcal{G B} \mathcal{W}_{\mathcal{T}}(\mathcal{A})$, then $a^{n} \in \mathcal{G B} \mathcal{W}_{\mathcal{T}}(\mathcal{A})$ for every $n \in \mathbb{N}$.

(iv) If $a \in \mathcal{G B B}_{\mathcal{T}}(\mathcal{A})$, then $a^{n} \in \mathcal{G B B}_{\mathcal{T}}(\mathcal{A})$ for every $n \in \mathbb{N}$.

(v) $\mathcal{G B W}_{\mathcal{T}}(\mathcal{A}) \backslash \mathcal{W}_{\mathcal{T}}(\mathcal{A}) \subseteq \mathcal{G B F}_{\mathcal{T}}(\mathcal{A}) \backslash \mathcal{F}_{\mathcal{T}}(\mathcal{A})$

(vi) $\mathcal{G B B}_{\mathcal{T}}(\mathcal{A}) \backslash \mathcal{B}_{\mathcal{T}}(\mathcal{A}) \subseteq \mathcal{G B F}_{\mathcal{T}}(\mathcal{A}) \backslash \mathcal{F}_{\mathcal{T}}(\mathcal{A})$

(vii) $\sigma_{\mathcal{G B} \mathcal{W}_{\mathcal{T}}}(a)=\bigcap_{c \in \mathcal{T}^{-1}(0)} \sigma_{\mathcal{K D}}(a+c)(a \in \mathcal{A})$.

(viii) $\sigma_{\mathcal{G B B}}(a)=\bigcap_{c \in \mathcal{T}^{-1}(0), a c=c a} \sigma_{\mathcal{K} \mathcal{D}}(a+c)(a \in \mathcal{A})$.

(ix) The sets $\sigma_{\mathcal{G B} \mathcal{W}_{\mathcal{T}}}(a)$ and $\sigma_{\mathcal{G B B}}(a)$ are closed $(a \in \mathcal{A})$.

Proof. (i). It follows from the fact that $\mathcal{B}^{\text {qnil }} \backslash \mathcal{B}^{\text {nil }} \subseteq \mathcal{B}^{\mathcal{K D}} \backslash \mathcal{B}^{\mathcal{D}}$.

(ii). Apply [17, Theorem 5.5].

(iii)-(iv). These statements can be proved using an argument similar to the one in Theorem 3.4(viii) and Theorem 3.4(ix), respectively.

(v)-(vi). Apply an argument similar to the one in Theorem 3.4(x)-(xi), using [17, Theorem 3.1] instead [28, Proposition 1(a)]. (vii)-(viii). These statements can be easily deduced.

(ix). Apply statements (vii) and (viii).

\section{Perturbations of B-Fredholm elements}

To prove the main results of this section, some preparation is needed.

Given $S \subseteq \mathcal{A}$, the commuting perturbation class of $S$, is the set

$$
P_{\text {comm }}(S)=\left\{a \in \mathcal{A}: S+_{\text {comm }}\{a\} \subset S\right\},
$$

where, if $H, K \subseteq \mathcal{A}$

$$
H+{ }_{\text {comm }} K=\{c+d:(c, d) \in H \times K, c d=d c\} .
$$


Let $\mathcal{A}$ and $\mathcal{B}$ be two unital Banach algebras and consider the homomorphism $\mathcal{T}: \mathcal{A} \rightarrow \mathcal{B}$. Given $K \subseteq \mathcal{B}$, it is not difficult to prove that

$$
\mathcal{T}^{-1}\left(P_{\text {comm }}(K)\right) \subseteq P_{\text {comm }}\left(\mathcal{T}^{-1}(K)\right) .
$$

In particular, $\mathcal{T}^{-1}\left(P_{\text {comm }}\left(\mathcal{B}^{\mathcal{D}}\right)\right) \subseteq P_{\text {comm }}\left(\mathcal{T}^{-1}\left(\mathcal{B}^{\mathcal{D}}\right)\right)=P_{\text {comm }}\left(\mathcal{B F}_{\mathcal{T}}(\mathcal{A})\right)$. Moreover, it is clear that $\mathcal{T}^{-1}\left(\operatorname{Poly}^{-1}(\{0\})\right)=\operatorname{Poly}^{-1}\left(\mathcal{T}^{-1}(0)\right)$. Also, if $K_{1}, K_{2} \subseteq$ $\mathcal{A}$ are such that $K_{1} \cap K_{2} \neq \emptyset$ then

$$
P_{\text {comm }}\left(K_{1}\right) \cap P_{\text {comm }}\left(K_{2}\right) \subseteq P_{\text {comm }}\left(K_{1} \cap K_{2}\right) .
$$

On the other hand, it is well known that $b \in \mathcal{A}^{\text {qnil }}$ if and only if for every $a \in \mathcal{A}$ which commutes with $b$ there is the equivalence:

$$
a \in \mathcal{A}^{-1} \Longleftrightarrow a+b \in \mathcal{A}^{-1}
$$

that is,

$$
a \notin \mathcal{A}^{-1} \Longleftrightarrow a+b \notin \mathcal{A}^{-1} \text {. }
$$

The equivalences (4.3) and (4.4) hold also if $\mathcal{A}^{-1}$ is replaced by $\mathcal{A}_{\text {left }}^{-1}$ or $\mathcal{A}_{\text {right }}^{-1}$. Consequently,

$$
\mathcal{A}^{\text {qnil }}=P_{\text {comm }}\left(\mathcal{A}^{-1}\right)=P_{\text {comm }}\left(\mathcal{A}_{\text {left }}^{-1}\right)=P_{\text {comm }}\left(\mathcal{A}_{\text {right }}^{-1}\right),
$$

and also,

$$
\mathcal{A}^{\text {qnil }}=P_{\text {comm }}\left(\mathcal{A} \backslash \mathcal{A}^{-1}\right)=P_{\text {comm }}\left(\mathcal{A} \backslash \mathcal{A}_{\text {left }}^{-1}\right)=P_{\text {comm }}\left(\mathcal{A} \backslash \mathcal{A}_{\text {right }}^{-1}\right) .
$$

In first place, a preliminary result is considered.

Proposition 4.1. Let $\mathcal{A}$ be a unital Banach algebra and consider an algeraic element $a \in \mathcal{A}$. Then, $a \in \mathcal{A}^{\text {qnil }}$ if and only if $a$ is nilpotent.

Proof. Every nilpotent element is quasinilpotent. On the other hand, if $a \in$ $\mathcal{A}^{\text {qnil }}$, then let $P \in \mathbb{C}[X]$ be the minimal polynomial such that $P(a)=0$. It is well known that $\sigma(a)=P^{-1}(\{0\})$. Since $\sigma(a)=\{0\}$, there must exist $k \in \mathbb{N}$ such that $P(X)=X^{k}$. Consequently, $a$ is nilpotent.

In the following theorem the commuting perturbation class of $\mathcal{A}^{\mathcal{D}}$ and $\mathcal{A}^{\mathcal{K D}}$ will be considered.

Theorem 4.2. Let $\mathcal{A}$ be a unital Banach algebra. Then,

(i) $\mathcal{A}^{\text {nil }} \subseteq P_{\text {comm }}\left(\mathcal{A}^{\mathcal{D}}\right) \subseteq \operatorname{Poly}^{-1}(\{0\})$.

(ii) $\mathcal{A}^{\text {qnil }} \subseteq P_{\text {comm }}\left(\mathcal{A}^{\mathcal{K D}}\right)$.

(iii) $\mathcal{A}^{\text {nil }} \subseteq P_{\text {comm }}\left(\mathcal{A}^{\mathcal{D}} \backslash \mathcal{A}^{-1}\right)$.

(iv) $\mathcal{A}^{\text {qnil }} \subseteq P_{\text {comm }}\left(\mathcal{A}^{\mathcal{K D}} \backslash \mathcal{A}^{-1}\right)$.

Proof. (i). Let $b \in \mathcal{A}^{\text {nil }}$ and $a \in \mathcal{A}^{\mathcal{D}}$ such that $a b=b a$. Since $b \in \mathcal{A}^{\mathcal{D}}$ and $b^{d}=0$, according to [29, Theorem 3], $a+b \in \mathcal{A}^{\mathcal{D}}$. In order to prove the remaining inclusion, suppose that $b \in P_{\text {comm }}\left(\mathcal{A}^{\mathcal{D}}\right)$. The elements $\lambda 1(=\lambda)$ are Drazin invertible and commute with $b$ for every $\lambda \in \mathbb{C}$. Therefore, $b+\lambda \in \mathcal{A}^{\mathcal{D}}$ for every $\lambda \in \mathbb{C}$. In particular, $\sigma_{\mathcal{D}}(b)=\emptyset$. Therefore, according to [6, Theorem 2.1], $b$ is algebraic.

(ii). It follows from [29, Theorem 8] and from the fact that $b^{D}=0$ if $b \in$ $\mathcal{A}^{\text {qnil }}$. 
(iii). Since $\mathcal{A}^{\text {nil }} \subseteq P_{\text {comm }}\left(\mathcal{A}^{\mathcal{D}}\right)$ and $\mathcal{A}^{\text {nil }} \subseteq \mathcal{A}^{\text {qnil }}=P_{\text {comm }}\left(\mathcal{A} \backslash \mathcal{A}^{-1}\right)$ (identity (4.5)), apply (4.2) to obtain $\mathcal{A}^{\text {nil }} \subseteq P_{\text {comm }}\left(\mathcal{A}^{\mathcal{D}} \cap\left(\mathcal{A} \backslash \mathcal{A}^{-1}\right)\right)=P_{\text {comm }}\left(\mathcal{A}^{\mathcal{D}} \backslash\right.$ $\left.\mathcal{A}^{-1}\right)$.

(iv). It follows from (ii), (4.5) and (4.2).

Corollary 4.3. Let $\mathcal{A}$ be a unital Banach algebra. Then

$$
\operatorname{Poly}^{-1}(\{0\}) \cap \mathcal{A}^{q n i l} \subseteq P_{\text {comm }}\left(\mathcal{A}^{\mathcal{D}}\right) .
$$

Proof. Apply Proposition 4.1 and Theorem 4.2 (i).

Remark 4.4. Let $\mathcal{A}$ be a unital Banach algebra. Then, the following statements hold.

(i) $\mathcal{A}^{\mathcal{K D}} \backslash \mathcal{A}_{\text {left }}^{-1}=\mathcal{A}^{\mathcal{K D}} \backslash \mathcal{A}_{\text {right }}^{-1}=\mathcal{A}^{\mathcal{K D}} \backslash \mathcal{A}^{-1}$.

(ii) $\mathcal{A}^{\mathcal{D}} \backslash \mathcal{A}_{\text {left }}^{-1}=\mathcal{A}^{\mathcal{D}} \backslash \mathcal{A}_{\text {right }}^{-1}=\mathcal{A}^{\mathcal{D}} \backslash \mathcal{A}^{-1}$.

(iii) $\mathcal{A}^{\mathcal{K D}} \cap \mathcal{A}_{\text {left }}^{-1}=\mathcal{A}^{\mathcal{K D}} \cap \mathcal{A}_{\text {right }}^{-1}=\mathcal{A}^{-1}$.

(iv) $\mathcal{A}^{\mathcal{D}} \cap \mathcal{A}_{\text {left }}^{-1}=\mathcal{A}^{\mathcal{D}} \cap \mathcal{A}_{\text {right }}^{-1}=\mathcal{A}^{-1}$.

To prove statement (i), note that $\mathcal{A}^{\mathcal{K D}} \backslash \mathcal{A}_{\text {left }}^{-1} \subseteq \mathcal{A}^{\mathcal{K D}} \backslash \mathcal{A}^{-1}$ and $\mathcal{A}^{\mathcal{K D}} \backslash$ $\mathcal{A}_{\text {right }}^{-1} \subseteq \mathcal{A}^{\mathcal{K D}} \backslash \mathcal{A}^{-1}$. Now suppose that $a \in \mathcal{A}^{\mathcal{K D}} \backslash \mathcal{A}^{-1}$. Then $0 \in$ iso $\sigma(a)$ ([17, Theorem 4.2]). It follows that 0 is a boundary point of $\sigma(a)$ and hence, it belongs to the left and also to the right spectrum of $a$. This implies that $a \notin \mathcal{A}_{\text {left }}^{-1}$ and $a \notin \mathcal{A}_{\text {right }}^{-1}$. In particular, $a \in \mathcal{A}^{\mathcal{K D}} \backslash \mathcal{A}_{\text {left }}^{-1} \cap \mathcal{A}^{\mathcal{K D}} \backslash \mathcal{A}_{\text {right }}^{-1}$. Therefore, $\mathcal{A}^{\mathcal{K D}} \backslash \mathcal{A}^{-1} \subseteq \mathcal{A}^{\mathcal{K D}} \backslash \mathcal{A}_{\text {left }}^{-1} \cap \mathcal{A}^{\mathcal{K D}} \backslash \mathcal{A}_{\text {right }}^{-1}$.

Statement (ii) can be proved using an argument simimilar to the one developed in the previous paragraph.

Statement (iii) (respectively statement (iv)) can be easily derived from statement (i) (respectively statement (ii)).

Next algebraic (nilpotent) elements will be characterized using the Drazin spectrum.

Theorem 4.5. Let $\mathcal{A}$ be a unital Banach algebra and consider $d \in \mathcal{A}^{\text {qnil }}$. Then the following statements are equivalent:

(i) The element $d$ is algebraic.

(ii) Given $a \in \mathcal{A}$, ad $=$ da implies that $\sigma_{\mathcal{D}}(a+d)=\sigma_{\mathcal{D}}(a)$.

Proof. If $d$ is algebraic, then according to Proposition 4.1, $d \in \mathcal{A}^{\text {nil }}$, which is equivalent to $-d \in \mathcal{A}^{\text {nil }}$. According to Corollary 4.3, $d,-d \in P_{\text {comm }}\left(\mathcal{A}^{\mathcal{D}}\right)$. Let $a \in \mathcal{A}$ such that $a d=d a$. If $\lambda \in \mathbb{C}$ is such that $\lambda \notin \sigma_{\mathcal{D}}(a)$, then $a-\lambda \in$ $\mathcal{A}^{\mathcal{D}}$, and since $d \in P_{\text {comm }}\left(\mathcal{A}^{\mathcal{D}}\right), a+d-\lambda \in \mathcal{A}^{\mathcal{D}}$. In particular, $\lambda \notin \sigma_{\mathcal{D}}(a+d)$. To prove the reverse, apply the same argument to $-d \in P_{\text {comm }}\left(\mathcal{A}^{\mathcal{D}}\right), a+d$ and $\lambda \notin \sigma_{\mathcal{D}}(a+d)$.

Conversely, if $a=0$, then $\sigma_{\mathcal{D}}(d)=\sigma_{\mathcal{D}}(0)=\emptyset$. However, according to 6 , Theorem 2.1], $d$ is algebraic.

In the following theorem the commuting perturbation class of (generalized) B-Fredholm elements will be considered.

Theorem 4.6. Let $\mathcal{A}$ and $\mathcal{B}$ be two unital Banach algebras and consider a (not necessarily continuous) homomorphism $\mathcal{T}: \mathcal{A} \rightarrow \mathcal{B}$. Then, (i) $\mathcal{N}_{\mathcal{T}}(\mathcal{A}) \subseteq P_{\text {comm }}\left(\mathcal{B F}_{\mathcal{T}}(\mathcal{A})\right) \subseteq T^{-1}\left(\operatorname{Poly}^{-1}(\{0\})\right.$ 
(ii) $\mathcal{R}_{\mathcal{T}}(\mathcal{A}) \subseteq P_{\text {comm }}\left(\mathcal{G B F}_{\mathcal{T}}(\mathcal{A})\right)$.

(iii) $\mathcal{N}_{\mathcal{T}}(\mathcal{A}) \subseteq P_{\text {comm }}\left(\mathcal{B F}_{\mathcal{T}}(\mathcal{A}) \backslash \mathcal{F}_{\mathcal{T}}(\mathcal{A})\right)$.

(iv) $\mathcal{R}_{\mathcal{T}}(\mathcal{A}) \subseteq P_{\text {comm }}\left(\mathcal{G B F}_{\mathcal{T}}(\mathcal{A}) \backslash \mathcal{F}_{\mathcal{T}}(\mathcal{A})\right)$.

Proof. (i). According to Theorem 4.2 (i) and (4.1),

$$
\mathcal{N}_{\mathcal{T}}(\mathcal{A}) \subseteq T^{-1}\left(P_{\text {comm }}\left(\mathcal{B}^{\mathcal{D}}\right)\right) \subseteq P_{\text {comm }}\left(T^{-1}\left(\mathcal{B}^{\mathcal{D}}\right)\right)=P_{\text {comm }}\left(\mathcal{B F}_{\mathcal{T}}(\mathcal{A})\right) .
$$

Let $a \in P_{\text {comm }}\left(\mathcal{B F}_{\mathcal{T}}(\mathcal{A})\right)$. Then for every $\lambda \in \mathbb{C}, a+\lambda \in \mathcal{B F}_{\mathcal{T}}(\mathcal{A})$, equivalently, $T(a)+\lambda \in \mathcal{B}^{\mathcal{D}}$. However, according to [6, Theorem 2.1], $T(a) \in \mathcal{B}$ is algebraic.

(ii)-(iv). Apply Theorem 4.2(ii)-(iv) and use an argument similar to the one in the proof of statement (i).

Corollary 4.7. Let $\mathcal{A}$ and $\mathcal{B}$ be two unital Banach algebras and consider a (not necessarily continuous) homomorphism $\mathcal{T}: \mathcal{A} \rightarrow \mathcal{B}$. Let $a \in \mathcal{B F}_{\mathcal{T}}(\mathcal{A})$ and $b \in \mathcal{N}_{\mathcal{T}}(\mathcal{A})$ such that $a b-b a \in \mathcal{T}^{-1}(0)$. Then, $a+b \in \mathcal{B F}_{\mathcal{T}}(\mathcal{A})$. In particular, $\sigma_{\mathcal{B F}_{\mathcal{T}}}(a+b)=\sigma_{\mathcal{B F}_{\mathcal{T}}}(a)$.

Proof. Apply Theorem 4.2 (i).

Corollary 4.8. Let $\mathcal{A}$ and $\mathcal{B}$ be two unital Banach algebras and consider a (not necessarily continuous) homomorphism $\mathcal{T}: \mathcal{A} \rightarrow \mathcal{B}$. If $a \in \mathcal{R}_{\mathcal{T}}(\mathcal{A})$ and $\mathcal{T}(a) \in \mathcal{B}$ is algebraic, then $a \in P_{\text {comm }}\left(\mathcal{B F}_{\mathcal{T}}(\mathcal{A})\right)$.

Proof. According to Corollary 4.3, $\mathcal{T}(a) \in P_{\text {comm }}\left(\mathcal{B}^{\mathcal{D}}\right)$. Therefore, $a \in \mathcal{T}^{-1}$ ( $\left.P_{\text {comm }}\left(\mathcal{B}^{\mathcal{D}}\right)\right) \subseteq P_{\text {comm }}\left(\mathcal{T}^{-1}\left(\mathcal{B}^{\mathcal{D}}\right)\right)=P_{\text {comm }}\left(\mathcal{B F}_{\mathcal{T}}(\mathcal{A})\right)$.

Under the same assumptions in Corollary [4.8, note that if $a \in \mathcal{A}$ is algebraic and $a \in \mathcal{R}_{\mathcal{T}}(\mathcal{A})$, then $\mathcal{T}(a) \in P_{\text {comm }}\left(\mathcal{B}^{\mathcal{D}}\right)$.

Let $\mathcal{A}$ and $\mathcal{B}$ be two unital Banach algebras and consider a (not necessarily continuous) homomorphism $\mathcal{T}: \mathcal{A} \rightarrow \mathcal{B}$. Recall that according to 31, Theorem 10.1], the following statements are equivalent:

(i) The element $d \in \mathcal{R}_{\mathcal{T}}(\mathcal{A})$.

(ii) If $a \in \mathcal{A}$ is such that $a d-d a \in \mathcal{T}^{-1}(0)$, then $\sigma_{\mathcal{F}_{\mathcal{T}}}(a)=\sigma_{\mathcal{F}_{\mathcal{T}}}(a+d)$.

(iii) If $a \in \mathcal{A}$ is such that $a d=a d$, then $\sigma_{\mathcal{F}_{\mathcal{T}}}(a)=\sigma_{\mathcal{F}_{\mathcal{T}}}(a+d)$.

(iv) $\sigma_{\mathcal{F}_{\mathcal{T}}}(d)=\{0\}$.

In the following theorem, a similar result for the B-Fredholm spectrum will be considered.

Theorem 4.9. Let $\mathcal{A}$ and $\mathcal{B}$ be two unital Banach algebras and consider a (not necessarily continuous) homomorphism $\mathcal{T}: \mathcal{A} \rightarrow \mathcal{B}$. Let $d \in \mathcal{R}_{\mathcal{T}}(\mathcal{A})$. Then, the following conditions are equivalent.

(i) $\mathcal{T}(d)$ is algebraic.

(ii) If $a \in \mathcal{A}$ is such that $a d-d a \in \mathcal{T}^{-1}(0)$, then $\left.\sigma_{\mathcal{B F}_{\mathcal{T}}}(a+d)=\sigma_{\mathcal{B F}_{\mathcal{T}}}(a)\right)$.

(iii) If $a \in \mathcal{A}$ is such that $a d=d a$, then $\left.\sigma_{\mathcal{B F}_{\mathcal{T}}}(a+d)=\sigma_{\mathcal{B F}_{\mathcal{T}}}(a)\right)$.

(iv) $\sigma_{\mathcal{B F}_{\mathcal{T}}}(d)=\emptyset$.

Proof. (i) $\Longrightarrow$ (ii). Apply Theorem 4.5 ,

(ii) $\Longrightarrow$ (iii). It is obvious.

(iii) $\Longrightarrow$ (iv). Consider $a=0$. Then, $\sigma_{\mathcal{B F}}(d)=\sigma_{\mathcal{B F}}(0)=\emptyset$.

(iv) $\Longrightarrow$ (i). Apply Theorem 3.2 (iv). 


\section{Perturbations of (Generalized) B-Fredholm elements With EQUAL SPECTRAL IDEMPOTENTS}

To prove the main results of this section, some prepartion is needed first.

Let $\mathcal{A}$ be a unital Banach algebra and consider $a \in \mathcal{A}^{\mathcal{K D}}$. Let $p=1-a^{D} a$. If $a \in \mathcal{A}^{-1}$, then $p=0$, but if $0 \in$ iso $\sigma(a), p$ is the spectral idempotent of $a$ corresponding to 0 and it will be written $p=a^{\pi}$. Note that $a p=p a$, $a p \in \mathcal{A}^{q n i l}, \quad a(1-p), a^{D} \in((1-p) \mathcal{A}(1-p))^{-1}$ and $a^{D}$ is the inverse of $(1-p) a$ in the algebra $(1-p) \mathcal{A}(1-p)([26])$.

Remark 5.1. Let $\mathcal{A}$ and $\mathcal{B}$ be two unital Banach algebras and consider a (non necessarily continuous) homomorphism $\mathcal{T}: \mathcal{A} \rightarrow \mathcal{B}$.

(a). Let $a \in \mathcal{A}$ such that $\mathcal{T}(a) \in \mathcal{B}^{\mathcal{K D}}$. Let $\mathcal{T}(a)^{\pi}=q$ and suppose that there exist $p \in \mathcal{A}^{\bullet}$ such that $\mathcal{T}(p)=q$ and $w \in(1-p) \mathcal{A}(1-p)$ such that $\mathcal{T}(w)=\mathcal{T}(a)^{D}=((1-q) \mathcal{T}(a)(1-q))^{-1} \in((1-q) \mathcal{B}(1-q))^{-1}$ ( [17, Theorem 4.2]). Then, it is not difficult to prove the following statements.

(i) $(1-p) a w=1-p+c_{1}$ and $w a(1-p)=1-p+c_{2}$, where $c_{i} \in \mathcal{T}^{-1}(0) \cap$ $(1-p) \mathcal{A}(1-p), i=1,2$.

(ii) If $w^{\prime} \in(1-p) \mathcal{A}(1-p)$ is such that $\mathcal{T}\left(w^{\prime}\right)=\mathcal{T}(a)^{D}$, then $w^{\prime}-w \in$ $\mathcal{T}^{-1}(0) \cap(1-p) \mathcal{A}(1-p)$.

(b). Suppose in addition that $\mathcal{T}: \mathcal{A} \rightarrow \mathcal{B}$ is surjective and has the lifting property and consider $a \in \mathcal{A}$ as before, i.e., $\mathcal{T}(a) \in \mathcal{B}^{\mathcal{K D}}$ and $\mathcal{T}(a)^{\pi}=q$. In particular, there exist $p \in \mathcal{A}^{\bullet}$ such that $\mathcal{T}(p)=q$ and $z \in \mathcal{A}$ such that $\mathcal{T}(a)^{D}=\mathcal{T}(z)$. However, since $\mathcal{T}(a)^{D} \in(1-q) \mathcal{B}(1-q)$, it is possible to choose $z \in(1-p) \mathcal{A}(1-p)$.

The results of Remark 5.1 will be used in what follows.

Proposition 5.2. Let $\mathcal{A}$ and $\mathcal{B}$ be two unital Banach algebras and consider $a$ (non necessarily continuous) homomorphism $\mathcal{T}: \mathcal{A} \rightarrow \mathcal{B}$. Let $a_{1} \in \mathcal{A}$ such that $\mathcal{T}\left(a_{1}\right) \in \mathcal{B}^{\mathcal{K D}}$ and $\mathcal{T}\left(a_{1}\right)^{\pi}=q$. Suppose that there exist $p \in \mathcal{A}^{\bullet}$ and $w_{1} \in(1-p) \mathcal{A}(1-p)$ such that $\mathcal{T}(p)=q$ and $\mathcal{T}\left(w_{1}\right)=\mathcal{T}\left(a_{1}\right)^{D}$. Let $a_{2} \in \mathcal{A}$ and define $z=1+\mathcal{T}\left(a_{1}\right)^{D} \mathcal{T}\left(a_{2}-a_{1}\right)$. Then, the following statements hold.

(i) The element $z \in \mathcal{B}^{-1}$ if and only if $p+w_{1} a_{2} \in \mathcal{F}_{\mathcal{T}}(\mathcal{A})$.

(ii) Suppose that $\mathcal{T}\left(a_{2}\right) \mathcal{T}\left(a_{1}\right)^{\pi}=\mathcal{T}\left(a_{1}\right)^{\pi} \mathcal{T}\left(a_{2}\right)$. Then, $z \in \mathcal{B}^{-1}$ if and only $p+w_{1} a_{2}(1-p) \in \mathcal{F}_{\mathcal{T}}(\mathcal{A})$.

Proof. (i). Note that $z \in \mathcal{B}^{-1}$ if and only if $1+\mathcal{T}\left(w_{1}\left(a_{2}-a_{1}\right)\right) \in \mathcal{B}^{-1}$. Since $\mathcal{T}\left(w_{1} a_{1}\right)=\mathcal{T}\left(w_{1} a_{1}(1-p)\right)=1-q$, necessary and sufficient for $z \in \mathcal{B}^{-1}$ is that $q+\mathcal{T}\left(w_{1} a_{2}\right) \in \mathcal{B}^{-1}$, which in turn is equivalent to $p+w_{1} a_{2} \in \mathcal{F}_{\mathcal{T}}(\mathcal{A})$. (ii). Since $\mathcal{T}\left(a_{1}\right)$ and $\mathcal{T}\left(a_{2}\right)$ commute with $q, z \in \mathcal{B}^{-1}$ if and only if $1-q+$ $\mathcal{T}\left(w_{1}\left(a_{2}-a_{1}\right)(1-p)\right) \in((1-q) \mathcal{B}(1-q))^{-1}$. Now, using an argument similar to one in the proof of statement $(\mathrm{i})$, it is not difficult to prove that $z \in \mathcal{B}^{-1}$ if and only if $p+w_{1} a_{2}(1-p) \in \mathcal{F}_{\mathcal{T}}(\mathcal{A})$.

In the following theorems, (generalized) B-Fredholm elements that have the same spectral idempotents relative to the homomorphism $\mathcal{T}$ will be characterized.

Theorem 5.3. Let $\mathcal{A}$ and $\mathcal{B}$ be two unital Banach algebras and consider a (non necessarily continuous) homomorphism $\mathcal{T}: \mathcal{A} \rightarrow \mathcal{B}$. Suppose in 
addition that $\mathcal{T}: \mathcal{A} \rightarrow \mathcal{B}$ is surjective and has the lifting property. Let $a_{1} \in \mathcal{G B F}_{\mathcal{T}}(\mathcal{A})$ and consider $p \in \mathcal{A}^{\bullet}$ such that $\mathcal{T}(p)=\mathcal{T}\left(a_{1}\right)^{\pi}$. Then, the following statements are equivalent.

(i) $a_{2} \in \mathcal{G B F}_{\mathcal{T}}(\mathcal{A})$ and $\mathcal{T}\left(a_{1}\right)^{\pi}=\mathcal{T}\left(a_{2}\right)^{\pi}$.

(ii) $p a_{2}(1-p)$ and $(1-p) a_{2} p \in \mathcal{T}^{-1}(0), p a_{2} p \in \mathcal{R}_{\mathcal{T}}(\mathcal{A})$ and $p+a_{2} \in \mathcal{F}_{\mathcal{T}}(\mathcal{A})$.

(iii) $p a_{2}(1-p)$ and $(1-p) a_{2} p \in \mathcal{T}^{-1}(0), p a_{2} p \in \mathcal{R}_{\mathcal{T}}(\mathcal{A})$ and $p+w_{1} a_{2}(1-p) \in$ $\mathcal{F}_{\mathcal{T}}(\mathcal{A})$, where $w_{1} \in(1-p) \mathcal{A}(1-p)$ is such that $\mathcal{T}\left(w_{1}\right)=\mathcal{T}\left(a_{1}\right)^{D}$.

(iv) $a_{2} \in \mathcal{G B F}_{\mathcal{T}}(\mathcal{A}), p+w_{1} a_{2} \in \mathcal{F}_{\mathcal{T}}(\mathcal{A})$ and $w_{1}=\left(p+w_{1} a_{2}\right) w_{2}+c$, where $w_{1}$ is as in statement (iii), $w_{2} \in \mathcal{A}$ is such that $\mathcal{T}\left(w_{2}\right)=\mathcal{T}\left(a_{2}\right)^{D}$ and $c \in \mathcal{T}^{-1}(0)$.

Proof. Apply Proposition 5.2 to [26, Theorem 2.2] and note the following fact (statement (iv)).

According to the proof of Proposition 5.2(i), the identity

$$
\mathcal{T}\left(a_{2}\right)^{D}=\left(1+\mathcal{T}\left(a_{1}\right)^{D} \mathcal{T}\left(a_{2}-a_{1}\right)\right)^{-1} \mathcal{T}\left(a_{1}\right)^{D}
$$

is equivalent to

$$
\left(q+\mathcal{T}\left(w_{1} a_{2}\right)\right) \mathcal{T}\left(w_{2}\right)=\mathcal{T}\left(w_{1}\right),
$$

which in turn is equivalent to $w_{1}=\left(p+w_{1} a_{2}\right) w_{2}+c, c \in \mathcal{T}^{-1}(0)$.

Remark 5.4. Under the same hypothesis of Theorem 5.3, let $a_{2} \in \mathcal{A}$ be such that $a_{2} \in \mathcal{G B F}_{\mathcal{T}}(\mathcal{A})$ and $\mathcal{T}\left(a_{1}\right)^{\pi}=\mathcal{T}\left(a_{2}\right)^{\pi}$.

(i) In particular, $w_{2} \in(1-p) \mathcal{A}(1-p)$ and

$$
w_{1}=\left(p+w_{1} a_{2}\right) w_{2}+c=w_{1} a_{2} w_{2}+c=w_{1}(1-p) a_{2}(1-p) w_{2}+c,
$$

where $c \in \mathcal{T}^{-1}(0) \cap(1-p) \mathcal{A}(1-p)$.

(ii) Consider again the identity

$$
\mathcal{T}\left(a_{2}\right)^{D}=\left(1+\mathcal{T}\left(a_{1}\right)^{D} T\left(a_{2}-a_{1}\right)\right)^{-1} \mathcal{T}\left(a_{1}\right)^{D} .
$$

It is not difficult to prove that

$$
\mathcal{T}\left(w_{2} a_{1}(1-p)\right) \mathcal{T}\left(w_{1} a_{2}(1-p)\right)=\mathcal{T}\left(w_{1} a_{2}(1-p)\right) \mathcal{T}\left(w_{2} a_{1}(1-p)\right)=1-q .
$$

However, since $z=q+\mathcal{T}\left(w_{1} a_{2}(1-p)\right.$ ) (Proposition 5.2(ii)), $z^{-1}=q+$ $\mathcal{T}\left(w_{2} a_{1}(1-p)\right)$.

In particular,

$$
\mathcal{T}\left(a_{2}\right)^{D}=\left(1+\mathcal{T}\left(a_{1}\right)^{D} T\left(a_{2}-a_{1}\right)\right)^{-1} \mathcal{T}\left(a_{1}\right)^{D}
$$

is equivalent to $w_{2}=w_{2} a_{1}(1-p) w_{1}+d, d \in \mathcal{T}^{-1}(0) \cap(1-p) \mathcal{A}(1-p)$.

In the next theorem B-Fredholm elements that have the same spectral idempotents relative to the homomorphism $\mathcal{T}$ will be characterized.

Theorem 5.5. Let $\mathcal{A}$ and $\mathcal{B}$ be two unital Banach algebras and consider a (non necessarily continuous) homomorphism $\mathcal{T}: \mathcal{A} \rightarrow \mathcal{B}$. Suppose in addition that $\mathcal{T}: \mathcal{A} \rightarrow \mathcal{B}$ is surjective and has the lifting property. Let $a_{1} \in \mathcal{B F}_{\mathcal{T}}(\mathcal{A})$ and consider $p \in \mathcal{A}^{\bullet}$ such that $\mathcal{T}(p)=\mathcal{T}\left(a_{1}\right)^{\pi}$. Then, the following statements are equivalent.

(i) $a_{2} \in \mathcal{B F}_{\mathcal{T}}(\mathcal{A})$ and $\mathcal{T}\left(a_{1}\right)^{\pi}=\mathcal{T}\left(a_{2}\right)^{\pi}$.

(ii) $p a_{2}(1-p)$ and $(1-p) a_{2} p \in \mathcal{T}^{-1}(0), p a_{2} p \in \mathcal{N}_{\mathcal{T}}(\mathcal{A})$ and $p+a_{2} \in \mathcal{F}_{\mathcal{T}}(\mathcal{A})$. 
(iii) $p a_{2}(1-p)$ and $(1-p) a_{2} p \in \mathcal{T}^{-1}(0), p a_{2} p \in \mathcal{N}_{\mathcal{T}}(\mathcal{A})$ and $p+w_{1} a_{2}(1-p) \in$ $\mathcal{F}_{\mathcal{T}}(\mathcal{A})$, where $w_{1} \in(1-p) \mathcal{A}(1-p)$ is such that $\mathcal{T}\left(w_{1}\right)=\mathcal{T}\left(a_{1}\right)^{d}$.

(iv) $a_{2} \in \mathcal{B F}_{\mathcal{T}}(\mathcal{A}), p+w_{1} a_{2} \in \mathcal{F}_{\mathcal{T}}(\mathcal{A})$ and $w_{1}=\left(p+w_{1} a_{2}\right) w_{2}+c$, where $w_{1}$ is as in statement (iii), $w_{2} \in \mathcal{A}$ is such that $\mathcal{T}\left(w_{2}\right)=\mathcal{T}\left(a_{2}\right)^{d}$ and $c \in \mathcal{T}^{-1}(0)$.

Proof. The same arguments in Remark 5.1 , Proposition 5.2 and Theorem 5.3 apply to the case of B-Fredholm elements using nilpotent elements instead of quasi-nilpotent elements. What is more, when considering Drazin invertible Banach algebra elements, statements similar to the ones in [26, Theorem 2.2] hold, if nilpotent elements instead of quasi-nilpotent elements are used.

Recall that in Theorem 3.5(ii) (respectively Theorem 3.4(vii)) conditions assuring that the product of two generalized B-Fredholm elements (respectively two B-Fredholm elements) is generalized B-Fredholm (respectively B-Fredholm) were given. In the following theorem more information concerning this problem will be given.

Theorem 5.6. Let $\mathcal{A}$ and $\mathcal{B}$ be two unital Banach algebras and consider a (non necessarily continuous) homomorphism $\mathcal{T}: \mathcal{A} \rightarrow \mathcal{B}$ such that $\mathcal{T}$ is surjective and has the lifting property. Let $a_{i} \in \mathcal{G B F}_{\mathcal{T}}(\mathcal{A}), i=1,2$, such that $\mathcal{T}\left(a_{1}\right)^{\pi}=\mathcal{T}\left(a_{2}\right)^{\pi}=q$ and $a_{1} a_{2}-a_{2} a_{1} \in \mathcal{T}^{-1}(0)$. Let $p \in \mathcal{A}^{\bullet}$ such that $\mathcal{T}(p)=q$. Then, $a_{1} a_{2} \in \mathcal{G B F}_{\mathcal{T}}(\mathcal{A}), \mathcal{T}\left(a_{1} a_{2}\right)^{\pi}=q$ and if $w_{1}, w_{2}$ and $w_{12} \in(1-p) \mathcal{A}(1-p)$ are such that $\mathcal{T}\left(w_{1}\right)=\mathcal{T}\left(a_{1}\right)^{D}, \mathcal{T}\left(w_{2}\right)=\mathcal{T}\left(a_{2}\right)^{D}$ and $\mathcal{T}\left(w_{12}\right)=\mathcal{T}\left(a_{1} a_{2}\right)^{D}$, then $w_{12}=w_{2} w_{1}+c, c \in \mathcal{T}^{-1}(0)$.

Proof. According to Theorem 3.5)(ii), $a_{1} a_{2} \in \mathcal{G B F}_{\mathcal{T}}(\mathcal{A})$. Moreover, since $\mathcal{T}\left(a_{1}\right), \mathcal{T}\left(a_{2}\right) \in \mathcal{B}^{\mathcal{K D}}$ and $\mathcal{T}\left(a_{1}\right) \mathcal{T}\left(a_{2}\right)=\mathcal{T}\left(a_{2}\right) \mathcal{T}\left(a_{1}\right)$, according to [17, Theorem 5.5], $\mathcal{T}\left(a_{1} a_{2}\right)^{D}=\mathcal{T}\left(a_{1}\right)^{D} \mathcal{T}\left(a_{2}\right)^{D}=\mathcal{T}\left(a_{2}\right)^{D} \mathcal{T}\left(a_{1}\right)^{D}$. Further, since $\mathcal{T}\left(a_{1}\right)^{\pi}=\mathcal{T}\left(a_{2}\right)^{\pi}=q$,

$$
\begin{aligned}
\mathcal{T}\left(a_{1} a_{2}\right)^{\pi} & =1-\mathcal{T}\left(a_{1} a_{2}\right) \mathcal{T}\left(a_{1} a_{2}\right)^{D}=1-\mathcal{T}\left(a_{1}\right) \mathcal{T}\left(a_{2}\right) \mathcal{T}\left(a_{2}\right)^{D} \mathcal{T}\left(a_{1}\right)^{D} \\
& =1-\mathcal{T}\left(a_{1}\right)(1-q) \mathcal{T}\left(a_{1}\right)^{D}=1-(1-q) \mathcal{T}\left(a_{1}\right) \mathcal{T}\left(a_{1}\right)^{D} \\
& =1-(1-q)(1-q)=1-(1-q) \\
& =q .
\end{aligned}
$$

Since $\mathcal{T}\left(a_{1} a_{2}\right)^{D}=\mathcal{T}\left(a_{2}\right)^{D} \mathcal{T}\left(a_{1}\right)^{D}, \mathcal{T}\left(a_{1} a_{2}\right)^{D}=\mathcal{T}\left(w_{2}\right) \mathcal{T}\left(w_{1}\right)$. Consequently, $w_{12}=w_{2} w_{1}+c, c \in \mathcal{T}^{-1}(0)$.

Acknowledgements. The first and third authors are supported by the Ministry of Education, Science and Technological Development, Republic of Serbia, grant no. 174007.

\section{REFERENCES}

[1] H. Baklouti, $\mathcal{T}$-Fredholm analysis and applications to operator theory, J. Math. Anal. Appl. 369 (2010), 283-289.

[2] M. Berkani, On a class of quasi-Fredholm operators, Integral Equations Operator Theory 34 (1999), 244-249. 
[3] M. Berkani, Index of B-Fredholm operators and generalization of a Weyl's theorem, Proc. Amer. Math. Soc. 130 (2002), 1717-1723.

[4] M. Berkani and M. Sarih, An Atkinson-type theorem for B-Fredholm operators, Studia Math. 148 (2001), 251-257.

[5] E. Boasso, Drazin spectra of Banach space operators and Banach algebra elements, J. Math. Anal. Appl. 359 (2009), 48-55.

[6] E. Boasso, The Drazin spectrum in Banach algebras, An Operator Theory Summer: Timisoara June 29-July 4 2010, International Book Series of Mathematical Texts, Theta Foundation, Bucharest, 2012, 21-28

[7] E. Boasso, Isolated spectral points and Koliha-Drazin invertible elements in quotient Banach algebras and homomorphism ranges, submitted; http://arxiv.org/abs/1403.3663.

[8] D. Djordjević, Regular and T-Fredholm elements in Banach algebras, Publ. Inst. Math. (Beograd) (N.S.) 56 (70) (1994), 90-94.

[9] M. P. Drazin, Pseudo-inverses in associative rings and semigroups, Amer. Math. Monthly 65 (1958), 506-514.

[10] J.J. Grobler and H. Raubenheimer, Spectral properties of elements in different Banach algebras, Glasgow Math. J. 33 (1991), 11-20.

[11] R. Harte, Fredholm theory relative to a Banach algebra homomorphism, Math. Z. 179 (1982), 431-436.

[12] R. Harte, Fredholm, Weyl and Browder theory, Proc. R. Ir. Acad. 85A (1985), 151-176.

[13] R. Harte, Regular boundary elements, Proc. Amer. Math. Soc. 99 (1987), 328-330.

[14] R. Harte, Fredholm, Weyl and Browder theory II, Proc. R. Ir. Acad. 91A (1991), 79-88.

[15] R. Harte and H. Raubenheimer, Fredholm, Weyl and Browder theory III, Proc. R. Ir. Acad. 95A (1995), 11-16.

[16] C. King, A note on Drazin inverses, Pacific J. Math. 70 (1977), 383-390.

[17] J. J. Koliha, A generalized Drazin inverse, Glasgow Math. J. 38 (1996), 367-381

[18] V. Kordula and V. Müller, On the axiomatic theory of spectrum, Studia Math. 119 (1996), 109-128.

[19] R. A. Lubansky, Koliha-Drazin invertibles form a regularity, Math. Proc. Roy. Ir. Acad. 107A (2007), 137-141.

[20] T. Mouton and H. Raubenheimer, Fredholm theory relative to two Banach algebra homomorphisms, Quaest. Math. 14 (1991), 371-382.

[21] H. du T. Mouton and H. Raubenheimer, More on Fredholm theory relative to a Banach algebra homomorphisms, Math. Proc. R. Ir. Acad. 93A (1993), 17-25.

[22] H. du T. Mouton, S. Mouton and H. Raubenheimer, Ruston elements and Fredholm theory relative to arbitrary homomorphisms, Quaest Math. 34 (2011), 341-359.

[23] V. Müller, On the Kato-decomposition of quasi-Fredholm and B-Fredholm operators, Preprint ESI 1013, Vienna, 2001.

[24] V. Müller, Spectral theory of linear operators and spectral systems in Banach algebras, Operator Theory, Advances and Applications 139, Birkhäuser Verlag, BaselBoston-Berlin, 2007.

[25] V. Rakočević, A note on regular elements in Calkin algebras, Collect. Math. 43 (1992), 37-42.

[26] V. Rakočević, Koliha-Drazin invertible operators and commuting Riesz perturbations, Acta Sci. Math. (Szeged) 68 (2002), 953-963.

[27] V. Rakočević, Koliha-Drazin inverse and perturbations, Rend. Circ. Mat. Palermo (2) Suppl. 73 (2004), 101-125.

[28] S. Roch, B. Silbermann, Continuity of generalized inverses in Banach algebras, Studia Math. 13 (1999), 197-227. 
[29] G.F. Zhuang, J.L. Chen, D.S. Cvetković-Ilić and Y.M. Wei, Additive property of Drazin invertibility of elements in a ring, Linear Multilinear Algebra 60 (2012), 903-910.

[30] S. Č. Živković-Zlatanović and R. Harte, On almost essentially Ruston elements of a Banach algebra, Filomat 24 (2010), 149-155.

[31] S. Č. Živković-Zlatanović, D. S. Djordjević and R. Harte, Ruston, Riesz and perturbation classes, J. Math. Anal. Appl. 389 (2012), 871-886.

[32] S. Č. Živković-Zlatanović, D. S. Djordjević and R. Harte, Polynomially Riesz perturbations, J. Math. Anal. Appl. 408 (2013), 442-451.

UNIVERSITY OF NIŠ, FACULTY O SCIENCES AND MATHEMATICS, VIŠEGRADSKA 33, P.O. BOX 224, 18000 NIŠ, SERBIA

E-mail address: milosCvetkovic83@gmail.com

E-mail address: enrico_odisseo@yahoo.it

UNIVERSITY OF NIŠ, FACULTY O SCIENCES AND MATHEMATICS, VIŠEGRADSKA 33, P.O. BOX 224, 18000 NIŠ, SERBIA

E-mail address: mladvlad@open.telekom.rs 